\title{
Comparison of therapeutic effects of different mesenchymal stem cells on rheumatoid arthritis in mice
}

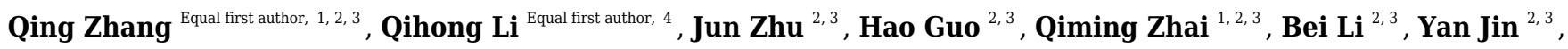 \\ Xiaoning He ${ }^{\text {Corresp., 2, } 3}$, Fang Jin Corresp. 1, 2, 3 \\ ${ }^{1}$ State Key Laboratory of Military Stomatology \& National Clinical Research Center for Oral Diseases \& Shaanxi Clinical Research Center for Oral Diseases, \\ Department of Orthodontics, School of Stomatology, The Fourth Military Medical University, Xi'an, Shaanxi, China \\ 2 State Key Laboratory of Military Stomatology \& National Clinical Research Center for Oral Diseases \& Shaanxi International Joint Research Center for \\ Oral Diseases, Center for Tissue Engineering, School of Stomatology, The Fourth Military Medical Universit, Xi'an, Shaanxi, China \\ 3 Xi'an Institute of Tissue Engineering and Regenerative Medicine, Xi'an, Shaanxi, China \\ 4 Department of Stomatology, the fifth Medical Centre, Chinese PLA General Hospital, Beijing, China \\ Corresponding Authors: Xiaoning He, Fang Jin \\ Email address: hxn_800222@163.com, jinfang@fmmu.edu.cn
}

Background: Rheumatoid arthritis (RA) is a chronic and nonspecific autoimmune disease (ADs), which leads to joint destruction and deformity. To investigate the potential of human mesenchymal stem cells (MSCs) as a new therapeutic strategy for patients with RA, we compared the therapeutic effects of bone marrow derived MSCs (BMSCs), umbilical cord derived MSCs (UCS), and stem cells derived from human exfoliated deciduous teeth (SHED) on collagen-induced arthritis (CIA) in mice. Methods: Twenty-four DBA/1 mice were infused with type II collagen to induce RA in the experimental model. MSC-treated mice were infused with UCS, BMSCs, and SHED, respectively. Bone erosion and joint destruction were measured by micro-computed tomographic (micro-CT) analysis and hematoxylin and eosin (HE) staining. The levels of tumor necrosis factor $\alpha$ (TNF- $\alpha)$ and interleukin-1 $(\mathrm{IL}-1 \beta$ ) were measured by immunohistochemistry and Enzyme-Linked Immunosorbent Assay (ELISA). Results: Systemic delivery of MSCs significantly improved the severity of the symptoms related to $\mathrm{CIA}$ to greater extent compared with the untreated control group. Micro-CT revealed reduced bone erosions in the metatarsophalangeal joints upon treatment with MSCs. Additionally, according to histologic evaluation, reduced synovitis and articular destruction were observed in MSC-treated groups. The levels of TNF- $\alpha$ and IL-1 $\beta$ in the serum and joints decreased with treatment by MSCs. Conclusion: Our findings suggest that systemic infusion of UCs, BMSCs, and SHED may significantly alleviate the effects of RA. The therapeutic effect of BMSCs was greater than that of SHED, while the UCS were shown to have the best therapeutic effect on CIA mice. In conclusion, compared with BMSCs and SHED, UCs may be a more suitable source of MSCs for the treatment of patients with RA. 
2 Comparison of Therapeutic Effects of Different

3 Mesenchymal Stem Cells on Rheumatoid Arthritis in

4 mice

6 Qing Zhang ${ }^{1,2,3^{*}}$, Qihong $\mathrm{Li}^{4 *}$, Jun Zhu ${ }^{2,3}$, Hao Guo ${ }^{2,3}$, Qiming Zhai ${ }^{1,2,3}$, Bei Li ${ }^{2,3}$, Yan Jin ${ }^{2,3}$,

7 Xiaoning $\mathrm{He}^{2,3}$ and Fang Jin ${ }^{1,2,3}$

$9{ }^{1}$ State Key Laboratory of Military Stomatology \& National Clinical Research Center for Oral

10 Diseases \& Shaanxi Clinical Research Center for Oral Diseases, Department of Orthodontics,

11 School of Stomatology, The Fourth Military Medical University, Xi'an, Shaanxi, China;

$122^{2}$ State Key Laboratory of Military Stomatology \& National Clinical Research Center for Oral

13 Diseases \& Shaanxi International Joint Research Center for Oral Diseases, Center for Tissue

14 Engineering, School of Stomatology, The Fourth Military Medical University, Xi'an, Shaanxi,

15 China;

$16{ }^{3}$ Xi' an Institute of Tissue Engineering and Regenerative Medicine, Xi'an, Shaanxi, China;

$17{ }^{4}$ Department of Stomatology, the fifth Medical Centre, Chinese PLA General Hospital, Beijing, 18 China.

19

$20 *$ These authors contributed equally to this work 
22 Corresponding Author:

23 Fang Jin ${ }^{1}$,

24 No.145 West Changle Road, Xi'an, Shaanxi, 710032, China

25 Email address: jinfang@fmmu.edu.cn

26 Xiaoning $\mathrm{He}^{2,3}$

27 No.145 West Changle Road, Xi'an, Shaanxi, 710032, China

28 Email address: $\underline{\text { hxn 800222@163.com }}$

\section{Abstract}

31 Background: Rheumatoid arthritis (RA) is a chronic and nonspecific autoimmune disease

32 (ADs), which leads to joint destruction and deformity. To investigate the potential of human 33 mesenchymal stem cells (MSCs) as a new therapeutic strategy for patients with RA, we compared

34 the therapeutic effects of bone marrow derived MSCs (BMSCs), umbilical cord derived MSCs

35 (UCs), and stem cells derived from human exfoliated deciduous teeth (SHED) on collagen-induced 36 arthritis (CIA) in mice.

37 Methods: Twenty-four DBA/1 mice were infused with type II collagen to induce RA in the experimental model. MSC-treated mice were infused with UCs, BMSCs, and SHED, respectively. Bone erosion and joint destruction were measured by micro-computed tomographic (micro-CT) analysis and hematoxylin and eosin (HE) staining. The levels of tumor necrosis factor $\alpha$ (TNF- $\alpha$ )

41 and interleukin-1 $\beta$ (IL-1 $\beta$ ) were measured by immunohistochemistry and Enzyme-Linked 42 Immunosorbent Assay (ELISA).

43 Results: Systemic delivery of MSCs significantly improved the severity of the symptoms related 44 to CIA to greater extent compared with the untreated control group. Micro-CT revealed reduced 
45 bone erosions in the metatarsophalangeal joints upon treatment with MSCs. Additionally,

46 according to histologic evaluation, reduced synovitis and articular destruction were observed in

47 MSC-treated groups. The levels of TNF- $\alpha$ and IL-1 $\beta$ in the serum and joints decreased with

48 treatment by MSCs.

49 Conclusion: Our findings suggest that systemic infusion of UCs, BMSCs, and SHED may

50 significantly alleviate the effects of RA. The therapeutic effect of BMSCs was greater than that of

51 SHED, while the UCs were shown to have the best therapeutic effect on CIA mice. In conclusion,

52 compared with BMSCs and SHED, UCs may be a more suitable source of MSCs for the treatment

53 of patients with RA.

54 Introduction

55 RA, a chronic and nonspecific inflammation, is characterized by recurrent, progressive joint pain 56 and swelling. It attacks joints throughout the entire body, which may lead to joint destruction and 57 eventual deformity (Scott, Wolfe $F \&$ Huizinga, 2010). Bone loss at the articular end, joint space 58 stenosis, and worm-like erosions on the articular or bone margin, are the typical X-ray features of 59 RA (Arnett et al., 1988). According to statistics from the World Health Organization, the global 60 incidence rate of RA is $0.5 \%-1 \%$ of the population and the disability rate over the course of 15 61 years is $61.3 \%$ (Smolen, Aletaha \& McInnes, 1988). RA not only causes a decline in patients' 62 physical function, quality of life, and social participation, but also brings a huge economic burden 63 to the families of these patients and society at large.

64

65 Currently, it is widely accepted that immune dysfunction plays a significant role in the 66 pathogenesis and course of RA (S.B.Abramson \& A.Amin, 1988). Characteristic of RA, TNF- $\alpha$ 67 causes acute and chronic inflammation of the synovium and ultimately leads to tissue destruction. 
68 TNF- $\alpha$ also induces the release of other cytokines, including IL-1, IL-6, and chemokines. TNF- $\alpha$

69 and IL-1 can not only change the function of synovial fibroblasts to cause them to secrete a variety

70 of inflammatory mediators, such as matrix metalloproteinase IL-6 and prostaglandin E, but can

71 also enable synovial macrophages to differentiate into osteoclasts and help activate osteoclasts to

72 absorb bone (Fox, 2000; Lam et al., 2000). Many nonsteroidal anti-inflammatory drugs, disease

73 modifying antirheumatic drugs, and glucocorticoids have been used to treat RA. However, these

74 drugs do not work on all patients. The drugs mentioned above present some shortcomings related

75 to the incidence of side effects and high recurrence rates of RA (Joensuu et al., 2015). Therefore,

76 it is important to develop a new and more effective therapy for RA.

78 For the last several years, mesenchymal stem cells (MSCs) have been widely studied and implemented as a new therapeutic tool for an increasing number of clinical diseases. MSC with strong immunomodulatory and anti-inflammatory effects can repair damaged tissues by regulating

81 the local environment through cellular interactions and via the secretion of multiple factors. These 82 characteristics make MSC an ideal tool for the treatment of autoimmune diseases (ADs), such as 83 RA (Maumus, Jorgensen \& Noël, 2013; Kastrinaki \& Papadaki, 2009). The therapeutic effect of MSC on RA is attributed to the regulation on immune cells and inflammatory cytokines involved

85 in the course of RA. MSCs inhibit the proliferation and activation of T-lymphocyte and B-cell by 86 secretion of cytokines (paracrine effect) and cell-cell direct contact effect (Di et al., 2002; 87 Soleymaninejadian, Pramanik \& Samadian, 2012; Mohammadzadeh et al., 2014; Franquesa et 88 al., 2015; Corcione et al., 2006; Schena et al., 2010). Meanwhile. MSCs could also transform M189 type pro-inflammatory macrophage into M2-type anti-inflammatory macrophage. Thus, the 90 expression of pro-inflammatory cytokines was reduced while the expression of pro-inflammatory 
91 cytokines was increased (Krisztián et al., 2009). According to previous studies, MSC infusion

92 through venous or intraperitoneal injection have shown positive effects on animals and human

93 patients with RA (Swart \& Wulffraat, 2014). Although clinical trials on MSC therapy in RA are

94 limited, the safety and effectiveness of this treatment measures in RA have been proved.

95 Intravenous infusions of allogeneic adipose-derived MSCs in 46 patients with active refractory

96 RA were in general well tolerated without evidence of toxicity over 3 months (Álvaro et al., 2017).

97 The treatment of intravenous infusion of allogeneic BMSCs or UCs into four RA patients who

98 were resistant to DMARDs was safe and resulted in partial clinical improvement (Liang et al.,

99 2012). Intravenous injection of UCs in addition to DMARDs induced a significant clinical

100 improvement in patients who had inadequate responses to traditional medication (Wang et al.,

101 2013). Although most animal studies and limited clinical trials proved the positive effects of MSC

102 in RA, few studies indicated that MSCs did not confer any benefit in RA (Schurgers et al., 2010)

103 or even aggravated RA (Chen et al., 2010), particularly when the cells were applied at a point

104 where inflammation is high (Djouad et al., 2005).

105

106 BMSCs, UCs, and SHED are the three most widely used MSC for treatment at present. All three

107 MSC populations above are able to be productively and safely isolated and purified. In the past,

108 BMSCs were considered the most common cell source for stem-cell-based therapy. However,

109 aspirating bone marrow is an invasive and painful procedure. In addition, there are some

110 limitations in using BMSCs due to the high degree of viral exposure and the significant decrease

111 in cell number and proliferation/differentiation capacity with the increasing age of the donor

112 (Stolzing et al., 2008; Rao \& Mattson, 2001; Wakitani, Saito \& Caplan, 1995; Young et al., 1998).

113 On the contrary, UCs and SHED, derived from discarded human tissues are able to be 
114 noninvasively isolated from umbilical cords and exfoliated deciduous teeth, respectively. And

115 compared to BMSCs, UCs and SHED present with better proliferation ability and greater potential

116 for differentiation were considered better cell sources for therapeutic applications (Al-toub et al.,

117 2013; Hunt, 2011; Nakamura S et al., 2009). Previous animal studies and clinical trials reported

118 BMSCs and UCs could have a curative effect on RA (Liu et al., 2010). But until now, there were

119 no reports on the curative effect of SHED on RA. There have also been no comparative analyses

120 on the therapeutic efficacy of these three MSC populations on RA.

121

122 In this paper, we not only explored the curative effects of SHED on RA, but also compared the 123 efficacy of BMSCs, UCs, and SHED on RA, expecting to provide a basis for the use of different 124 MSCs in the clinical treatment of RA or other ADs.

\section{Materials \& Methods}

\section{Animals}

128 DBA/1 mice were purchased from Beijing Vital River Laboratory in Beijing, China. All mice were

129 8-weeks-old and were housed four to five per cage under specific pathogen-free conditions $\left(22^{\circ} \mathrm{C}\right.$, $13050 \%-55 \%$ humidity, and $12 \mathrm{~h}$ light/12 h dark cycles) with ready access to food and water. All 131 animal-handling procedures and experimental protocols performed were approved by the

132 guidelines set forth by the Animal Care Committee of the Fourth Military Medical University, 133 Xi'an, China (2018-kq-007).

\section{Isolation and culture of MSCs}

135 The collection of human tissues was approved by the Ethics Committee of the Fourth Military 136 Medical University (IRB-REV-2013-002). The umbilical cords were cut into segments $(2-3 \mathrm{~cm})$ 
137 after rinsed several times in sterile phosphate-buffered saline (PBS). Cord vessels, including 2

138 arteries and 1 vein, were removed. Then the umbilical cords were cut into small pieces $\left(0.5-1 \mathrm{~cm}^{3}\right)$

139 and placed directly into $10 \mathrm{~cm}$ culture dishes for culture expansion in basal culture medium. After

$1405 \mathrm{~d}$, non-adherent cells were discarded and adherent cells were continued to culture (KATHY et al.,

141 2003; Xinyu et al., 2018; Shang et al., 2017). Dental pulps from human exfoliated deciduous teeth

142 were cut into small pieces and digested with collagenase I (Gibco, Grand Island, NY, USA) for 1

$143 \mathrm{~h}$ at $37^{\circ} \mathrm{C}$. Single-cell suspensions were cultured in basal culture medium (Masako et al., 2003;

144 Kun et al., 2018). Cells were purified from the bone marrow aspirates of the iliac crest using the

145 Percoll density gradient centrifugation method and cultured in basal culture medium (Liao et al.,

146 2017; Wenjia et al., 2018; Wenjia et al., 2018). The basal culture medium was composed of $\alpha$ -

147 MEM medium (Gibco, Grand Island, NY, USA), 10\% FBS (Sijiqing, Hangzhou, China), 2 mm L-

148 glutamine (Invitrogen, Carlsbad, CA), $100 \mathrm{U} / \mathrm{mL}$ penicillin, and $100 \mathrm{U} / \mathrm{mL}$ streptomycin

149 (Invitrogen, Carlsbad, CA). Single-cell suspensions were equally seeded in $10 \mathrm{~cm}$ dishes (Thermo,

150 Suzhou, China) and initially maintained in an atmosphere of $5 \% \mathrm{CO} 2$ at $37^{\circ} \mathrm{C}$. The medium was

151 changed every 3 days until the adherent cells were $80-90 \%$ confluent. Then MSCs were passaged

152 after digestion with $0.25 \%$ trypsin. MSCs at passage three were used for the tests in this research.

153 Evaluation of surface markers by flow cytometry

154 Eight cell surface markers (CD105, CD73, CD90, CD45, CD34, CD14, CD19, and HLA-DR)

155 were evaluated to identify MSCs according to ISCT criteria (M et al., 2006). MSCs were counted

156 to ensure each cell suspension had more than $1 \times 10^{6}$ cells. The cells were incubated with human

157 anti-CD105 (PE), anti-CD73 (FITC), anti-CD90 (PE), anti-CD45(PE), anti-CD34(PE), anti-

158 CD14(FITC), anti-CD19 (APC), and anti-HLA-DR (FITC) (all from eBioscience, USA), 
159 respectively for $30 \mathrm{~min}$ at room temperature. The cells were then washed and suspended for flow

160 cytometry analysis.

161 Osteogenic differentiation assay

162 MSCs were seeded in six-well plates and cultured in basal medium for 24 hours. Then, cells were

163 cultured for 28 days with osteogenic medium, containing $10 \mathrm{mM} \beta$-glycerophosphate (Sigma-

164 Aldrich, USA), $50 \mu \mathrm{g} / \mathrm{ml}$ ascorbic acid (Sigma-Aldrich) and $100 \mathrm{nM}$ dexamethasone (Sigma-

165 Aldrich). The medium was refreshed every 3 days. The cells washed twice with PBS to remove 166 medium and then fixed with 4\% paraformaldehyde (Sigma-Aldrich). Then cells were washed 167 gently three times with ddH2O and stained with 1\% Alizarin Red (Sigma-Aldrich). Photographs 168 were taken by an inverted microscope (Olympus, Japan).

169 Adipogenic differentiation assay

170 MSCs were seeded in six-well plates and cultured in basal medium for 24 hours. Then, cells were

171 cultured for 14 days with adipogenic medium, containing $0.5 \mathrm{mM}$ isobutylmethylxanthine (MP,

172 USA), $10 \mu \mathrm{g} / \mathrm{ml}$ insulin, $0.5 \mathrm{mM}$ dexamethasone (MP, USA) and $60 \mathrm{mM}$ indomethacin (MP,

173 USA). The medium was refreshed every 3 days. The cells washed twice with PBS to remove

174 medium and then fixed with 4\% paraformaldehyde (Sigma-Aldrich). Then cells were washed 175 gently three times with ddH2O and stained with Oil Red O (Sigma-Aldrich). Photographs were 176 taken by the inverted optical microscope (Olympus, Japan).

177 Establishment of experimental CIA model and treatment by infusion of MSCs

178 Twenty-four DBA/1 mice were randomly assigned to four experimental groups: CIA mice (CIA),

179 CIA mice infused with UCs (UC), CIA mice infused with BMSCs (BMSC), and CIA mice infused

180 with SHED (SHED). CIA model was induced as described previously (David, Kary \& Edward,

181 2007). Briefly, DBA/1 mice received an injection into the base of the tail with $100 \mu \mathrm{g}$ of type II 
182 collagen (CII) (Chondrex; MD Biosciences) emulsified in Freund's complete adjuvant. 15 days

183 later, they received booster injections of $100 \mu \mathrm{g}$ of type II collagen in Freund's incomplete

184 adjuvant. On day $30,1 \times 10^{6}$ MSCs dissolved in $200 \mu$ PBS per mouse were administered via tail

185 vein injection to three groups (UC, BMSC and SHED) and an equal volume of PBS was

186 administered to the control group (CIA).

187 Disease severity score

188 Each paw was evaluated for a period of every three days after the infusion of MSCs and were

189 scored individually for the severity of arthritis using a previously described scoring system (Chen

190 et al., 2018; Maogen et al., 2013). No evidence of erythema and swelling received a score of 0 ,

191 erythema and mild swelling confined to the tarsals or metatarsals received a score of 1 , erythema

192 and moderate swelling of tarsal and the metatarsal or tarsal and ankle joints received a score of 2,

193 erythema and severe swelling extending from the ankle to metatarsal joints received a score of 3 ,

194 and erythema and severe swelling encompassing the ankle, foot, and digits, or ankylosis of the

$195 \operatorname{limb}$ received a score of 4 . Four paws scores added up to a total score per mouse.

$196 \boldsymbol{E L I S A}$

197 Mice were sacrificed 30 days after MSC infusion. Mouse blood was collected at eye socket and 198 serum was harvested after centrifugated at $3000 \mathrm{rpm}$ for $20 \mathrm{~min}$. TNF- $\alpha$ and IL-1 $\beta$ levels in mice 199 serum were measured using ELISA kits (Yanhui biotechnology, China) according to the 200 manufacturer's instructions.

\section{Micro-CT analysis}

202 Mice hind limbs were cut off after blood collection. Micro-CT scans of the hind limbs were 203 performed using an explore Locus SP micro CT scanner (GE, America) with the following 204 scanning parameters: scanning resolution: $14 \mathrm{um}$; rotation angle: $360^{\circ}$; tube voltage: $80 \mathrm{kV}$; tube 
205 current, $80 \mathrm{uA}$; exposure time, 2960ms; everage frame: 4; and pixel combination: $1 \times 1$. The

206 software program Micview V2.1.2 and ABA were used to create a 3D reconstruction and to

207 analyze the bone. The measured area of bone volume was set with a length of $1 \mathrm{~mm}$ in the distal

208 and proximal direction from the center of each metatarsophalangeal joint (Chen et al., 2018). The

209 mean bone volume of the second to fourth metatarsal and phalangeal bones were compared.

\section{Histological analysis}

211 Hind limbs were decalcified in 17\% EDTA for 15 days after being fixed for $48 \mathrm{~h}$ in $4 \%$

212 paraformaldehyde at $4^{\circ} \mathrm{C}$. Then hind limbs were embedded in paraffin and serially sectioned into

$2133 \mu \mathrm{m}$ thick paraffin sections. The sections were stained with haematoxylin-eosin (Leica

214 Biosystems) using standard protocols. Sections were observed under the light microscope (DM6B;

215 Leica). The extent of synovitis, pannus formation, and bone/cartilage destruction was determined

216 using a graded scale, as follows: no signs of inflammation received a score of 0 , mild inflammation

217 with hyperplasia of the synovial lining without cartilage destruction received a score of 1 ,

218 increasing degrees of inflammatory cell infiltration and cartilage/bone destruction received a score

219 of 2-4 (Chen et al., 2013) (Maogen et al., 2013).

220 Immunohistochemistry

221 The paraffin sections were incubated with primary antibodies TNF- $\alpha$ (1:200; Abcam) and IL-1 $\beta$

222 (1:200; Abcam). Secondary antibodies (1:1000) were purchased from Vector Laboratories. The

223 stained sections were observed using the light microscope (DM6B; Leica Microsystems,

224 Heerbrugg, Switzerland). The photographs were evaluated by Image J (Media Cybernetics, USA)

225 from three randomly selected views of each specimen. Quantification of the number of positively

226 stained cells or percentages of the positively stained area over the total area was performed using 
227 the Image J (National Institute of Health, Bethesda, MD, USA) software from three randomly

228 selected views of each specimen.

229 Statistical analysis

230 All numerical data are expressed as mean \pm standard deviation (SD) of six mice per group from

231 three independent experiments. Statistical analyses were performed using the Prism software

232 (GraphPad, La Jolla, CA). Statistical differences were assessed by one-way ANOVA for 233 comparison among multiple groups and Tukey's multiple comparisons test for comparison 234 between two groups. $\mathrm{P}<0.05$ was considered significant.

\section{Results}

\section{Isolation and characterization of MSCs}

237 Purified MSCs were successfully obtained from bone marrow, umbilical cords, and dental pulps 238 provided by donors. All the three MSC populations revealed positive expression ( $\geq 99 \%$ positive) 239 of CD105, CD73, CD90, and negative expression ( $\leq 1 \%$ positive) of CD45, CD34, CD14, CD19 240 and HLA-DR (Fig. 1A). Osteogenic and adipogenic differentiation assays were performed to 241 investigate the differentiation potential of MSCs. After osteogenic induction for 28 days, 242 mineralized nodules stained with Alizarin red were observed. (Fig. 1B). After culturing in 243 adipogenesis inducing medium for 14 days, the MSCs were found to form lipid droplets by staining 244 with Oil Red O (Fig. 1C). Based on the above results, the isolated MSCs met the ISCT criteria: 245 adhering to plastic, expressing specific surface antigen and having multipotent differentiation 246 potential (M et al., 2006).

\section{MSC treatment alleviate RA to different extent}

248 We successfully established the CIA model on mice and treated the mice with $1 \times 10^{6}$ MSC (Fig. 249 2A). The severity of RA was evaluated based on disease severity score, expression of inflammation 
250 cytokines, Micro-CT analysis and histological analysis. We recorded the severity score every three

251 days after MSC treatment. The mice treated with UCs showed the most obvious decline in their

252 severity score. However, the mice treated with BMSCs and SHED showed a lesser rate of decline

253 in their severity score (Fig. 2B). The severity of RA was determined with Micro-CT analysis by

254 measuring the shape and volume of bone (Fig. 3). Cartilage and bone destruction and inflammation

255 in mice ankles were revealed in CIA mice. Specifically, the joint surfaces had a rough and irregular

256 articular appearance with a diffuse lesion pattern on the articular surface (Fig. 3A). However, the

257 number of lesions were lower in MSCs treated mice (Figs. 3B, 3C, 3D). According to micro-CT

258 image quantitative analyses, mice treated with UCs presented with the highest bone volume.

259 Compared with those treated with BMSCs, the mice treated with SHED presented with a lower

260 bone volume (Fig. 3E). Cartilage destruction and inflammation in mice ankles were determined

261 by histopathological examination (Fig. 4). In CIA mice, the destruction of cartilage and bony

262 structures were observed on the articular surfaces of the joint. In addition, synovial hyperplasia

263 and inflammatory cell infiltration were observed on the articular surface of the joints from CIA

264 mice (Fig. 4A). Mice treated with UCs showed less bone/cartilage destruction and inflammatory

265 cell infiltration than the BMSCs and SHED treatment group (Figs. 4B, 4C, 4D). BMSCs and SHED

266 infusion could also reduce bone/cartilage destruction and inflammatory cell infiltration in CIA

267 mice, although BMSCs were more effective than SHED (Fig. 4E). According to previous studies,

268 changes in the expression of the inflammatory cytokines were observed in the joints and serum of

269 RA patients (Fox, 2000; Lam et al., 2000; Noh et al., 2009; Darrieutort et al., 2014). This led to

270 the investigation of the effect of MSC on the inhibition of the pro-inflammatory factors that are

271 most closely related to RA. The three kinds of MSC treatments effectively inhibited the expression

272 levels of TNF- $\alpha$ and IL-1 $\beta$ compared with the no treatment group (Fig. 5). UCs infusion reduced 
273 the TNF- $\alpha$ and IL- $1 \beta$ expression levels in joints and serum. Compared with UCs, the infusion of

274 BMSCs showed a higher level in the expression of TNF- $\alpha$ and IL-1 $1 \beta$. The expressions of TNF- $\alpha$ 275 and IL-1 $\beta$ were higher after SHED infusion compared with the infusions of BMSCs and UCs (Fig. 276 5).

277

278 Discussion

279 According to previous studies, tail vein infusion, intraperitoneal infusion, and intra-articular 280 implantation were three widely used methods to inject MSCs into mice. Tail vein infusion allows 281 stem cells to circulate more quickly and efficiently with the blood to the whole body, including 282 the damaged tissue. Therefore, MSCs infused through tail vein could effectively reduce systemic 283 inflammatory responses by paracrine effects, such as cell-cell interaction and cellular component 284 transfer. Furthermore, tail vein infusion is more similar to the infusion method used in clinical treatment. Take all the above advantages into consideration, tail vein infusion was used in this 286 study.

287 MSC, lacking major histocompatibility II and several costimulatory molecules, are relatively 288 immune-privileged, or at least are hypoimmunogenic, and have the ability to escape immune 289 recognition. In the last several years, MSCs were shown to modulate the innate and adaptive immunity (Glennie, Soeiro \& Dyson 2005; Sato et al., 2007). These cells may inhibit the function 291 of the major immune cell populations, including dendritic cells, T cells, B cells, and natural killer cells. Meanwhile, the immunomodulatory and anti-inflammatory properties of MSCs have been tested in a variety of animal models and have been applied in specific clinical settings (Schüle \& possible for MSCs to be applied in clinical treatments. Previous animal studies and clinical trials 
296 reported that MSCs may have a curative effect on some kinds of autoimmune diseases (Liang et 297 al., 2016).

298 The immunomodulatory ability of stem cells from different sources is controversial. UCs were 299 considered to have better immunosuppressive effect on T cells, B cells, and NK cells than BMSCs 300 (Najar et al., 2010; Li et al., 2014; Yoo et al., 2009; Ribeiro et al., 2013). Until now, there have

301 been few reports on the therapeutic effects of SHED on autoimmune diseases. SHED were reported 302 to have similar characteristics for immune regulation to MSCs (Alipour et al., 2013; Yamaza et 303 al., 2010). Furthermore, with advantages of outstanding proliferation capability, abundant cell

304 supply, and painless stem cell collection, SHED were indicated to be a desirable option as a cell source for therapeutic applications (Nakamura $S$ et al., 2009). For these reasons above, the immunomodulatory functions of SHED were verified. In this study, we noticed the strong

307 immunosuppressive ability of UCs in RA. The difference of immunosuppressive capabilities of 308 different MSCs was consistent with the previous reports (Najar et al., 2010; Li et al., 2014; Yoo 309 et al., 2009; Ribeiro et al., 2013; Alipour et al., 2013). The differences in immunomodulatory capacity may be due to that the three MSC populations come from different tissues (Ribeiro et al.,

311 2013). The weaker systemic regulatory ability of SHED may be due to their development from the 312 neural crest, which mainly differentiates into nerve cells or local tissue cells of the head.

314 In the course of RA, the dynamic balance of M1/M2 type macrophages was broken, leading to the 315 increase of M1-type macrophage, which releases proinflammatory cytokines, such as TNF- $\alpha$ and

316 IL-1 $\beta$. MSCs could transform M1-type pro-inflammatory macrophage into M2-type anti317 inflammatory macrophage through paracrine effects, thereby suppressing the inflammatory 318 response (Krisztián et al., 2009). IL-1 $\beta$ and TNF- $\alpha$ have been widely recognized as the dominant 
319 proinflammatory cytokines of RA. TNF- $\alpha$ plays an important role in local synovial inflammation,

320 pannus formation, and the tissue damage of RA (Bradley, 2008). Previous study suggests that

321 TNF- $\alpha$ inhibitors can obviously reduce the disease activity in RA patients and improve the

322 corresponding clinical symptoms (Hyrich et al., 2004). It has been recognized that high levels of

323 IL-1 $\beta$ have been detected in the serum and joint fluid of RA patients. Its level was closely related

324 to the activity and histopathological characteristics of the disease, such as synovial hyperplasia 325 and leukocyte infiltration (Noh et al., 2009; Darrieutort et al., 2014). To verify the effect of MSCs

326 on the expression of inflammatory cytokines closely associated with CIA pathogenesis, TNF- $\alpha$

327 and IL-1 $\beta$ levels in joints and serum were determined. The levels of IL-1 $\beta$ and TNF- $\alpha$ were 328 increased by CIA induction and decreased by treatment with MSCs. The decreases of these 329 inflammatory markers in the joints were consistent with those in the serum. Thus, systemic 330 injection of MSCs could relieve both systemic and joint inflammation. Our results indicate that 331 systemic injection of UCs may be the most appropriate new treatment for ADs.

\section{Conclusions}

333 In this study, we compared the therapeutic effects of BMSCs, UCs and SHED on mice with CIA.

334 Specifically, the therapeutic efficacy of UCs is better than BMSCs while that of BMSCs is better

335 than SHED on RA, according to the extent of reducing bone resorption, joint destruction, and 336 inflammatory factor expression. In studying the source of MSCs and their corresponding curative 337 effect, UCs are a better choice than BMSCs and SHED in clinical treatment on ADs, or at least on 338 RA.

339 Acknowledgements

340 References 
341 Alipour R, Adib M, Masoumi Karimi M, Hashemi-Beni B, Sereshki N. 2013. Comparing the

342 immunoregulatory effects of stem cells from human exfoliated deciduous teeth and bone

343 marrow-derived mesenchymal stem cells. Iranian Journal of Allergy Asthma and Immunology

$344 \quad$ 12(4):331-44.

345 Al Jumah MA, Abumaree MH. 2012. The immunomodulatory and neuroprotective effects of

346 mesenchymal stem cells (MSCs) in experimental autoimmune encephalomyelitis (EAE): a

347 model of multiple sclerosis (MS). International Journal of Molecular Sciences 13(7):9298-9331

348 DOI: 10.3390/ijms13079298.

349 Al-toub M, Almusa A, Almajed M, Al-Nbaheen M, Kassem M, Aldahmash A, Alajez NM.

350 2013. Pleiotropic effects of cancer cells secreted factors on human stromal (mesenchymal) stem

351 cells. Stem Cell Research \& Therapy 4(5):114 DOI:10.1186/scrt325.

352 Álvaro-Gracia JM, Jover JA, García-Vicuña R, Carreño L, Alonso A, Marsal S, Blanco F,

353 Martínez-Taboada VM, Taylor P, Martín-Martín C, DelaRosa O, Tagarro I, Díaz-

354 González F. 2017. Intravenous administration of expanded allogeneic adipose-derived

355 mesenchymal stem cells in refractory rheumatoid arthritis (Cx611): results of a multicentre,

356 dose escalation, randomised, single-blind, placebo-controlled phase Ib/IIa clinical trial. Annals

357 of the Rheumatic Diseases. 76(1):196-202. DOI: 10.1136/annrheumdis-2015-208918.

358 Arnett FC, Edworthy SM, Bloch DA, McShane DJ, Fries JF, Cooper NS, Healey LA, Kaplan

359 SR, Liang MH, Luthra HS. 1988. The American Rheumatism Association 1987 revised

360 criteria for the classification of rheumatoid arthritis. Arthritis \& Rheumatism 31(3):315-24

$361 \quad$ PMID: 3358796.

362 Bradley JR. 2008. TNF-mediated inflammatory disease. The Journal of Pathology 214(2):149-60

363 DOI: $10.1002 /$ path.2287. 
364 Brand DD, Latham KA, Rosloniec EF. 2007. Collagen-induced arthritis. Nature Protocols 365 2(5):1269-75 DOI: 10.1007/springerreference_37998.

366 Chen B, Hu J, Liao L, Sun Z, Han Q, Song Z, Zhao RC. 2010. Flk-1+ mesenchymal stem cells 367 aggravate collagen-induced arthritis by up-regulating interleukin-6. CLINICAL AND 368 EXPERIMENTAL IMMUNOLOGY. 159(3):292-302. DOI: $\quad 10.1111 / \mathrm{j} .1365-$ 369 2249.2009.04069.x.

370

371

372

373

374

375

376

377

378

379

380

381

382

383

384

385

Chen M, Su W, Lin X, Guo Z, Wang J, Zhang Q, Brand D, Ryffel B, Huang J, Liu Z, He X, Le AD, Zheng SG. 2013. Adoptive transfer of human gingiva-derived mesenchymal stem cells ameliorates collagen-induced arthritis via suppression of Th1 and Th17 cells and enhancement of regulatory $\mathrm{T}$ cell differentiation. Arthritis \& Rheumatology 65(5):1181-93 DOI: 10.1002/art.37894.

Chen W, Wang J, Xu Z, Huang F, Qian W Ma J, Wee HB, Lewis GS, June RR, Schafer PH, Lin J, Zheng SG. 2018. Apremilast Ameliorates Experimental Arthritis via Suppression of Th1 and Th17 Cells and Enhancement of CD4+Foxp3+ Regulatory T Cells Differentiation. Frontiers in Immunology 9:1662. DOI: 10.3389/fimmu.2018.01662.

Corcione A, Benvenuto F, Ferretti E, Giunti D, Cappiello V, Cazzanti F, Risso M, Gualandi F, Mancardi GL, Pistoia V, Uccelli A. 2006. Human mesenchymal stem cells modulate Bcell functions. Blood. 107(1):367-72. DOI: 10.1182/blood-2005-07-2657.

David D Brand, Kary A Latham, Edward F Rosloniec. 2007. Collagen-induced arthritis. NATURE PROTOCOLS. 2(5):1269-75. DOI: 10.1038/nprot.2007.173.

Darrieutort-Laffite C, Boutet MA, Chatelais M, Brion R, Blanchard F, Heymann D, Le Goff B. 2014. IL-1 $\beta$ and TNF $\alpha$ promote monocyte viability through the induction of GM-CSF 
expression by rheumatoid arthritis synovial fibroblasts. Mediators of Inflammation

387

388

389

390

391

392

393

394

395

396

397

398

399

400

401

402

403

404

405

406

407

408 2014:241840 DOI: $10.1155 / 2014 / 241840$.

David D Brand, Kary A Latham, Edward F Rosloniec. 2007. Collagen-induced arthritis. NATURE PROTOCOLS. 2(5):1269-75. DOI: 10.1038/nprot.2007.173.

Di Nicola M1, Carlo-Stella C, Magni M, Milanesi M, Longoni PD, Matteucci P, Grisanti S, Gianni AM. 2002. Human bone marrow stromal cells suppress T-lymphocyte proliferation induced by cellular or nonspecific mitogenic stimuli. Blood. 99(10):3838-3843.

Djouad F, Fritz V, Apparailly F, Louis-Plence P, Bony C, Sany J, Jorgensen C, Noël D. 2005. Reversal of the immunosuppressive properties of mesenchymal stem cells by tumor necrosis factor alpha in collagen-induced arthritis. ARTHRITIS \& RHEUMATISM. 52(5):1595-603. DOI: 10.1002/art.21012.

Fox DA. 2000. Cytokine blockade as a new strategy to treat rheumatoid arthritis: inhibition of tumor necrosis factor. Archives of Internal Medicine 160(4):437-44 DOI:10.1001/archinte.160.4.437.

Franquesa M, Mensah FK, Huizinga R, Strini T, Boon L, Lombardo E, DelaRosa O, Laman JD, Grinyó JM, Weimar W, Betjes MG, Baan CC, Hoogduijn MJ. 2015. Human adipose tissue-derived mesenchymal stem cells abrogate plasmablast formation and induce regulatory B cells independently of T helper cells. Stem Cells. 33(3):880-91. DOI: $10.1002 /$ stem. 1881 .

Glennie S, Soeiro I, Dyson PJ. 2005. Bone marrow mesenchymal stem cells induce division arrest anergy of activated T cells. Blood 105(7):2821-2827 DOI: 10.1182/blood-2004-09-3696.

Hunt CJ. 2011. Cryopreservation of human stem cells for clinical application: a review. Transfusion Medicine and Hemotherapy 38(2):107-123 DOI: 10.1159/000326623. 
409 Hyrich KL, Silman AJ, Watson KD, Symmons DP. 2004. Anti-tumour necrosis factor alpha

410 therapy in rheumatoid arthritis: an update on safety. Annals of the Rheumatic Diseases 411 63(12):1538-1543 DOI: 10.1136/ard.2004.024737.

412 Joensuu JT, Huoponen S, Aaltonen KJ, Konttinen YT, Nordström D, Blom M. 2015. The 413 cost-effectiveness of biologics for the treatment of rheumatoid arthritis: a systematic review. 414 PLoS One 10(3): e0119683 DOI: 10.1371/journal.pone.0119683.

415 Kastrinaki MC, Papadaki HA. 2009. Mesenchymal stromal cells in rheumatoid arthritis:

416 biological properties and clinical applications. Current Stem Cell Research \& Therapy 4(1): 61$417 \quad 69$ DOI: $10.2174 / 157488809787169084$.

418 KATHY E. MITCHELL, MARK L. WEISS, BRIANNA M. MITCHELL, PHILLIP 419 MARTIN, DUANE DAVIS, LOIS MORALES, BRYAN HELWIG, MARK BEERENSTRAUCH, KHALIL ABOU-EASA, TAMMI HILDRETH, DERYL TROYER. 2003. Matrix Cells from Wharton's Jelly Form Neurons and Glia. Stem Cells. 21(1):50-60. DOI: 10.1634/stemcells.21-1-50.

Krisztián Németh, Asada Leelahavanichkul, Peter S T Yuen, Balázs Mayer, Alissa

424 Parmelee, Kent Doi, Pamela G Robey, Kantima Leelahavanichkul, Beverly H Koller, 425 Jared M Brown, Xuzhen Hu, Ivett Jelinek, Robert A Star, Éva Mezey.2009. Bone marrow 426 stromal cells attenuate sepsis via prostaglandin E2-dependent reprogramming of host 427 macrophages to increase their interleukin-10 production. Nat Med. 15(1): 42-49 DOI:10.1038/nm.1905.

429 Lam J, Takeshita S, Barker JE, Kanagawa O, Ross FP, Teitelbaum SL. 2000. TNF-alpha 430 induces osteoclastogenesis by direct stimulation of macrophages exposed to permissive levels 
431 of RANK ligand. The Journal of Clinical Investigation 106:1481 1488 DOI: $432 \quad 10.1172 / \mathrm{JCI} 11176$.

433 Liao L, Shi B, Chang H, Su X, Zhang L, Bi C, Shuai Y, Du X, Deng Z, Jin Y. 2017. Heparin 434 improves BMSC cell therapy: Anticoagulant treatment by heparin improves the safety and 435 therapeutic effect of bone marrow-derived mesenchymal stem cell cytotherapy. Theranostics. $436 \quad 7(1): 106-116$. DOI: 10.7150/thno.16911.

437 Liang J, Li X, Zhang H, Wang D, Feng X, Wang H, Hua B, Liu B, Sun L. 2012. Allogeneic 438 mesenchymal stem cells transplantation in patients with refractory RA. Clinical 439 Rheumatology. 31(1):157-61. DOI: 10.1007/s10067-011-1816-0.

440 Liang J, Wang D, Dominique F, Sun L. 2016. Mesenchymal stem cells for treating autoimmune 441 diseases: The Chinese experience from lab to clinics. Current Research in Translational $442 \quad$ Medicine 64(2):115-20 DOI: 10.1016/j.retram.2016.04.007.

443 Liu Y, Mu R, Wang S, Long L, Liu X, Li R, Sun J, Guo J, Zhang X, Guo J, Yu P, Li C, Liu 444 X, Huang Z, Wang D, Li H, Gu Z, Liu B, Li Z. 2010. Therapeutic potential of human 445 umbilical cord mesenchymal stem cells in the treatment of rheumatoid arthritis. Arthritis 446 Research \& Therapy 12(6):R210 DOI: 10.1186/ar3187.

447 Li X, Bai J, Ji X, Li R, Xuan Y, Wang Y. 2014. Comprehensive characterization of four different 448 populations of human mesenchymal stem cells as regards their immune properties, proliferation 449 and differentiation. International Journal of Molecular Medicine 34(3):695-704 DOI: $450 \quad 10.3892 /$ ijmm.2014.1821.

451 Maogen Chen, Wenru Su, Xiaohong Lin, Zhiyong Guo, Julie Wang, Qunzhou Zhang,

452 David Brand, Bernhard Ryffel, Jiefu Huang, Zhongmin Liu, Xiaoshun He, Anh D. Le, 453 and Song Guo Zheng. 2013. Adoptive transfer of human gingiva-derived mesenchymal stem 
454 cells ameliorates collagen-induced arthritis via suppressing Th1 and Th17 and enhancing

455 regulatory T cell differentiation. Arthritis \& Rheumatology. 65(5): 1181-1193. DOI:

$456 \quad$ 10.1002/art.37894.

457 Masako Miura, Stan Gronthos, Mingrui Zhao, Bai Lu, Larry W. Fisher, Pamela Gehron

458 Robey, Songtao Shi. 2003. SHED: Stem cells from human exfoliated deciduous teeth.

459 Proceedings of the National Academy of Sciences of the United States of America. 100(10):

460 5807-5812. DOI: 10.1073/pnas.0937635100.

461 Maumus M1, Jorgensen C, Noël D. 2013. Mesenchymal stem cells in regenerative medicine

462 applied to rheumatic diseases: role of secretome and exosomes. Biochimie 95(12):2229-2234

463 DOI: 10.1016/j.biochi.2013.04.017.

464 Mohammadzadeh A, Pourfathollah AA, Shahrokhi S, Hashemi SM, Moradi SL, Soleimani M. 2014.

465 Immunomodulatory effects of adipose-derived mesenchymal stem cells on the gene expression of major

466 transcription factors of $\mathrm{T}$ cell subsets. International Immunopharmacology.20(2):316-21. DOI:

$467 \quad$ 10.1016/j.intimp.2014.03.003.

468 Nakamura S, Yamada Y, Katagiri W, Sugito T, Ito K, Ueda M. 2009. Stem cell proliferation

469 pathways comparison between human exfoliated deciduous teeth and dental pulp stem cells by 470 gene expression profile from promising dental pulp. Journal of Endodontics 35(11):1536-42

471 DOI: $10.1016 /$ j.joen.2009.07.024.

472 Najar M, Raicevic G, Boufker HI, Fayyad Kazan H, De Bruyn C, Meuleman N, Bron D,

473 Toungouz M, Lagneaux L. 2010. Mesenchymal stromal cells use PGE2 to modulate activation

474 and proliferation of lymphocyte subsets: Combined comparison of adipose tissue, Wharton's

475 Jelly and bone marrow sources. Cellular Immunology 264(2):171-9 DOI:

$476 \quad$ 10.1016/j.cellimm.2010.06.006. 
477 Noh EM, Kim JS, Hur H, Park BH, Song EK, Han MK, Kwon KB, Yoo WH, Shim IK, Lee

478 SJ, Youn HJ, Lee YR. 2009. Cordycepin inhibits IL-1beta-induced MMP-1 and MMP-3

479 expression in rheumatoid arthritis synovial fibroblasts. Rheumatology (Oxford) 48(1):45-48

480 DOI: $10.1093 /$ rheumatology/ken417.

481 Rao MS, Mattson MP. 2001. Stem cells and aging: expanding the possibilities. Mechanisms of 482 Ageing and Development 122(7):713-34 DOI: 10.1016/s0047-6374(01)00224-X.

483 Ribeiro A, Laranjeira P, Mendes S, Velada I, Leite C, Andrade P, Santos F, Henriques A, 484 Grãos M, Cardoso CM, Martinho A, Pais M, da Silva CL, Cabral J, Trindade H, Paiva A. 485 2013. Mesenchymal stem cells from umbilical cord matrix, adipose tissue and bone marrow 486 exhibit different capability to suppress peripheral blood B, natural killer and T cells. Stem Cell 487 Research \& Therapy 4(5):125 DOI: 10.1186/scrt336. 4(5):125.

488 Sato K, Ozaki K, Oh I, Meguro A, Hatanaka K, Nagai T, Muroi K, Ozawa K. 2007. Nitric 489 oxide plays a critical role in suppression of T-cell proliferation by mesenchymal stem cells. 490 Blood 109(1):228-234 DOI: 10.1182/blood-2006-02-002246.

491 S.B.Abramson, A.Amin. 2002. Blocking the effects of IL-1 in rheumatoid arthritis protects bone 492 and cartilage. Rheumatology 41(9):972-80 DOI: 10.1093/rheumatology/41.9.972.

493 Schena F, Gambini C, Gregorio A, Mosconi M, Reverberi D, Gattorno M, Casazza S,

494 Uccelli A, Moretta L, Martini A, Traggiai E. 2010. Interferon- $\gamma$-dependent inhibition of B

495 cell activation by bone marrow-derived mesenchymal stem cells in a murine model of

496 systemic lupus erythematosus. ARTHRITIS \& RHEUMATISM. 62(9):2776-86. DOI:

$497 \quad 10.1002 / \operatorname{art} .27560$. 
498 Schüle S, Berger A. 2015. Mesenchymal stromal cells in the treatment of graft-versus-host

499 disease: where do we stand. Bundesgesundheitsblatt Gesundheitsforschung Gesundheitsschutz.

500 58(11-12):1265-1273 DOI: 10.1007/s00103-015-2244-0.

501 Schurgers E, Kelchtermans H, Mitera T, Geboes L, Matthys P. 2010. Discrepancy between

502 the in vitro and in vivo effects of murine mesenchymal stem cells on T-cell proliferation and

503 collagen-induced arthritis. Arthritis Research \& Therapy. 12(1): R31. DOI: 10.1186/ar2939.

504 Scott DL, Wolfe F, Huizinga TW. 2010. Rheumatoid arthritis. The Lancet 376(9746): 1094-1108

505 DOI: $10.1016 / \mathrm{S} 0140-6736(10) 60826-4$.

506 Smolen JS, Aletaha D, McInnes IB. 2016. Rheumatoid arthritis. The Lancet 388(10055):2023-

5072038 DOI: 10.1016/S0140-6736(16)30173-8.

508 Soleymaninejadian E, Pramanik K, Samadian E. 2012. Immunomodulatory properties of

509 mesenchymal stem cells: cytokines and factors. American Journal of Reproductive

510 Immunology. 67(1):1-8. DOI: 10.1111/j.1600-0897.2011.01069.x.

511 Stolzing A, Jones E, McGonagle D, Scutt A. 2008. Age-related changes in human bone marrow-

512 derived mesenchymal stem cells: consequences for cell therapies. Mechanisms of Ageing and

513 Development 129(3):163-73 DOI: 10.1016/j.mad.2007.12.002.

514 Swart JF, Wulffraat NM. 2014. Mesenchymal stromal cells for treatment of arthritis. Best

515 Practice \& Research Clinical Rheumatology 28(4):589-603 DOI: 10.1016/j.berh.2014.10.023.

516 Wang L, Wang L, Cong X, Liu G, Zhou J, Bai B, Li Y, Bai W, Li M, Ji H, Zhu D, Wu M,

517 Liu Y. 2013. Human umbilical cord mesenchymal stem cell therapy for patients with active

518 rheumatoid arthritis: safety and efficacy. Stem Cells and Development. 22(24):3192-202.

519 DOI: $10.1089 / \mathrm{scd} .2013 .0023$. 
520 Yamaza T, Kentaro A, Chen C, Liu Y, Shi Y, Gronthos S, Wang S, Shi S. 2010.

521 Immunomodulatory properties of stem cells from human exfoliated deciduous teeth. Stem Cell

522 Research \& Therapy 1(1):5 DOI: 10.1186/scrt5.

523 Yoo KH, Jang IK, Lee MW, Kim HE, Yang MS, Eom Y, Lee JE, Kim YJ, Yang SK, Jung

524 HL, Sung KW, Kim CW, Koo HH. Comparison of immunomodulatory properties of

525 mesenchymal stem cells derived from adult human tissues. Cellular Immunology 259(2):150-6

526 DOI: $10.1016 /$ j.cellimm.2009.06.010. 
Figure 1

Characterization of hUCMSCs, hBMSCs and hSHED.

(A) Flow cytometric analysis of ex-vivo-expanded hUCMSCs, hBMSCs and hSHED. All the three MSC populations revealed positive expression of CD105, CD90, CD73, and negative expression of CD45, CD34, CD14, CD19 and HLA-DR. (B) After osteogenic induction for 28 days, hUCMSCs, hBMSCs and hSHED formed mineralized nodules stained by Alizarin Red. (C) After culturing in adipogenesis inducing medium for 14 days, hUCMSCs, hBMSCs and hSHED were found to form lipid droplets stained with Oil Red 0.

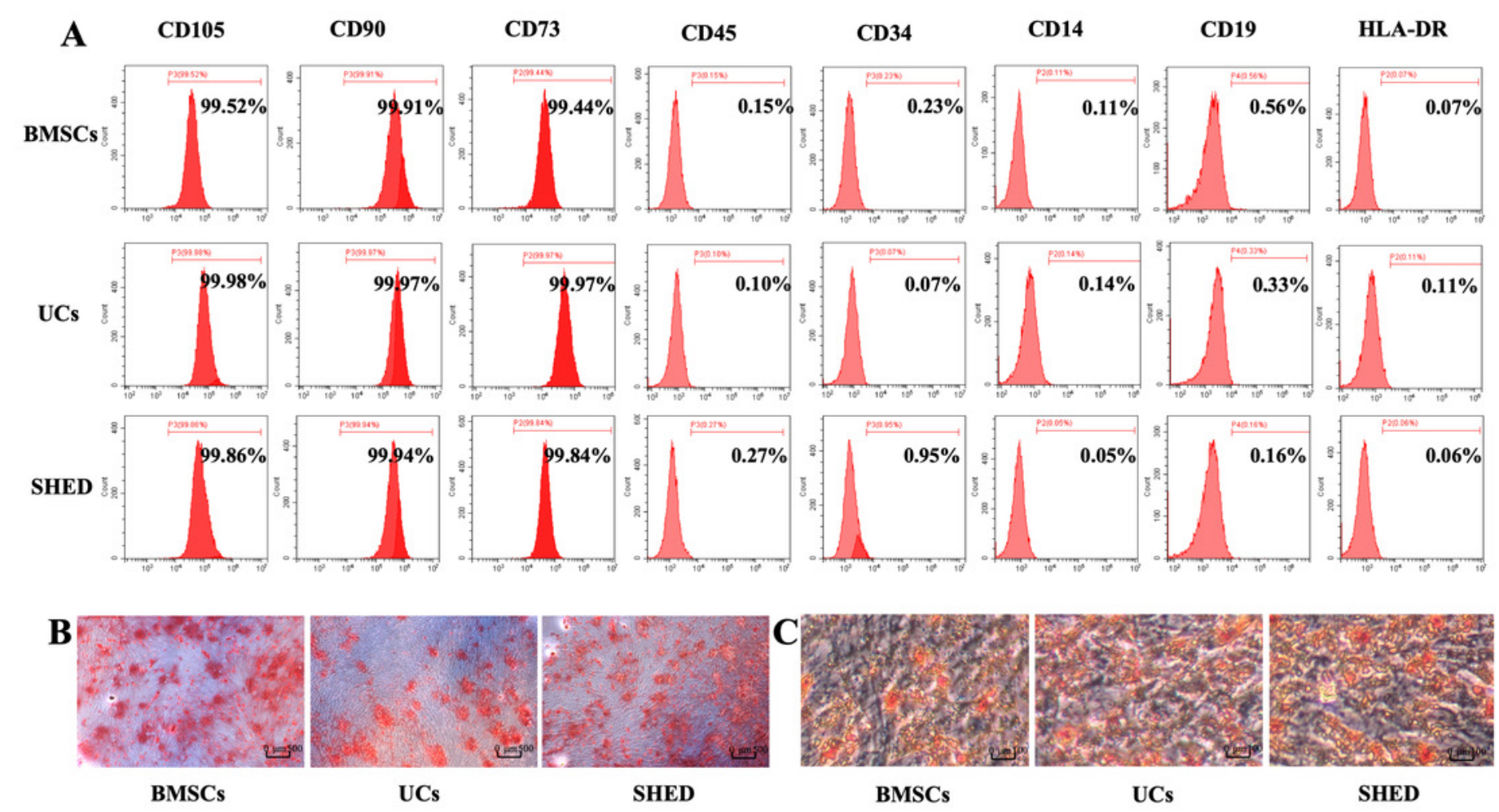


Figure 2

MSC treatment alleviate RA to different extents.

(A) Schema indicating of the study design. Type II collagenwas injected into mice twice via tail vein at $-4 \mathrm{w}$ and $-2 \mathrm{w}$ respectively. $1 \times 10^{6} \mathrm{MSCs}$ was infused into mice via tail vein at $0 \mathrm{w}$. All the mice were sacrificed at $4 \mathrm{w}$. (B) Disease severity score of 4 groups. The data indicate the mean \pm SD of six mice per group from three independent experiments. The data were analyzed using two-way ANOVA for comparison among multiple groups and Tukey's multiple comparisons test for comparison between two groups $(P<0.05)$.
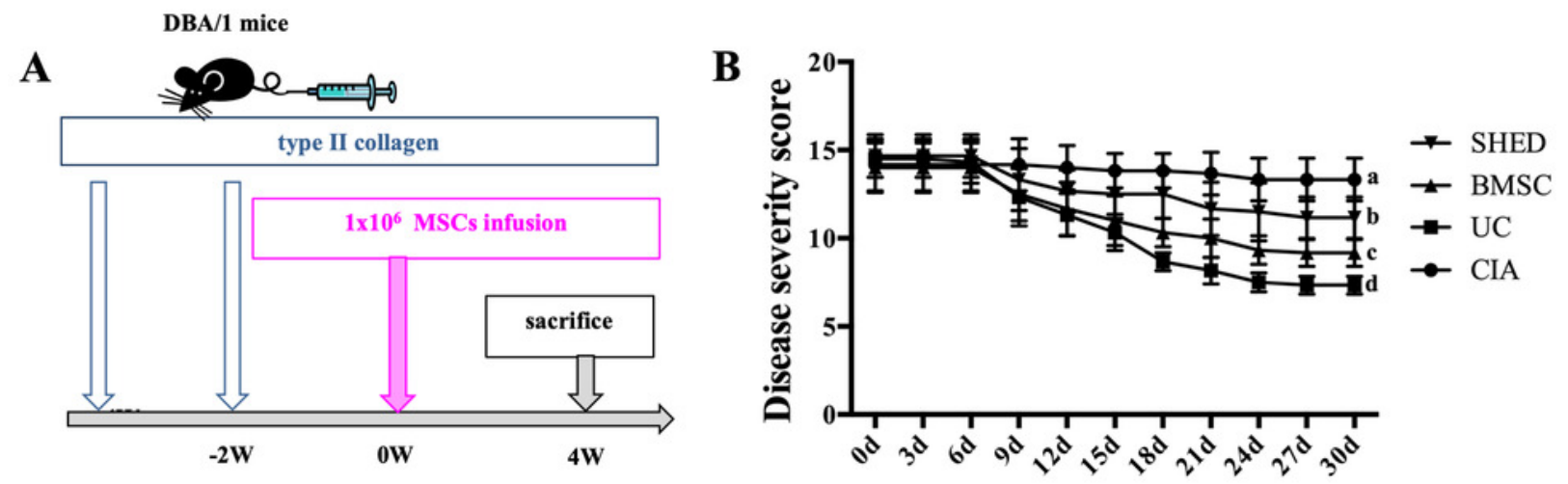


\section{Figure 3}

MSC treatment prevented bone erosion in CIA mice.

The bone volume measured area were set with $1 \mathrm{~mm}$ length in the distal and the proximal direction from the center of each metatarsophalangeal joint. The mean bone volume of second to fourth metatarsal and phalangeal bones were compared. (A) Bone erosion is observed in the CIA mice. (B) UC treatment reduced bone loss to the greatest degree. (C) BMSC treatment reduced bone loss to some extent. (D) SHED hadthe least treatment effect on reducing bone loss. (E) The mean bone volume of each group was evaluated and presented in column. The data indicate the mean \pm SD of six mice per group from three independent experiments. The data were analyzed using one-way ANOVA for comparison among multiple groups and Tukey's multiple comparisons test for comparison between two groups $(* * \mathrm{P}<0.01, * * * \mathrm{P}<0.001)$. 


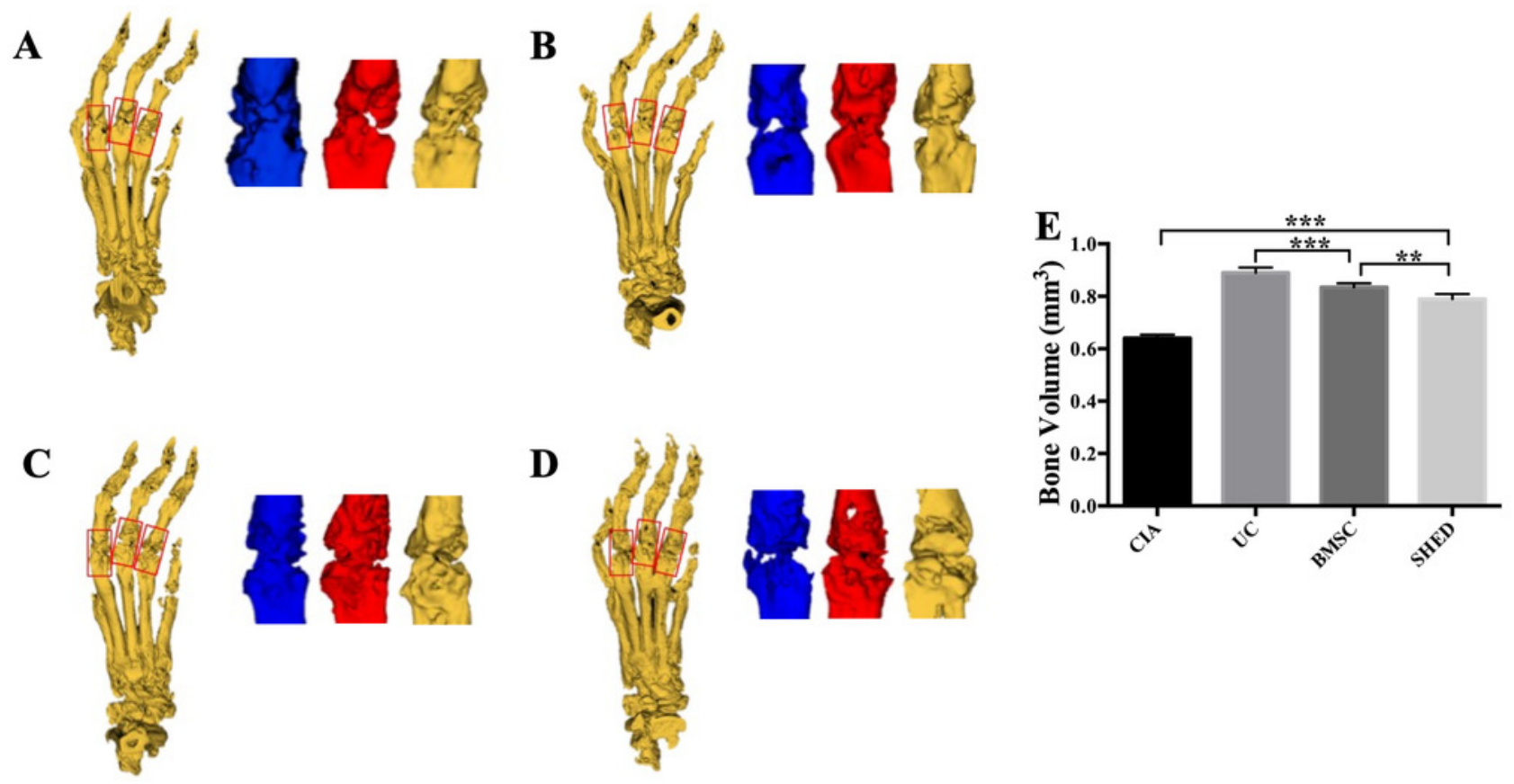




\section{Figure 4}

Histopathological examination of hind ankle joints of mice.

(A)Joint destruction is observed in the CIA mice. (B) UC treatmentreduced synovitis, pannus, erosion and inflammatory cells infiltration to the greatest degree. (C) BMSC treatmentreduced synovitis, pannus, erosion and inflammatory cells infiltration to some extent. (D) SHEDhadthe least treatment effect on reducing synovitis, pannus, erosion and inflammatory cells infiltration. (E) The histological score of each group was evaluated and presented in column. Scale bar, $75 \mu \mathrm{m}$. The data indicate the mean \pm SD of six mice per group from three independent experiments. The data were analyzed using one-way ANOVA for comparison among multiple groups and Tukey's multiple comparisons test for comparison between two groups $(* \mathrm{P}<0.05, * * \mathrm{P}<0.01)$. 
A

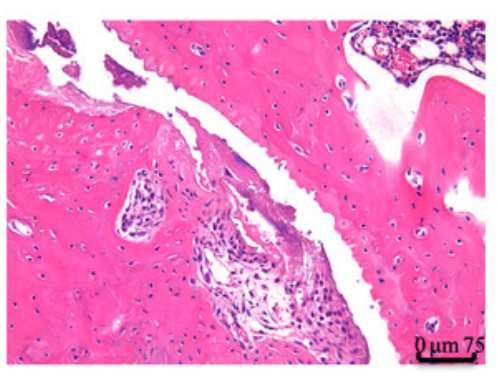

C

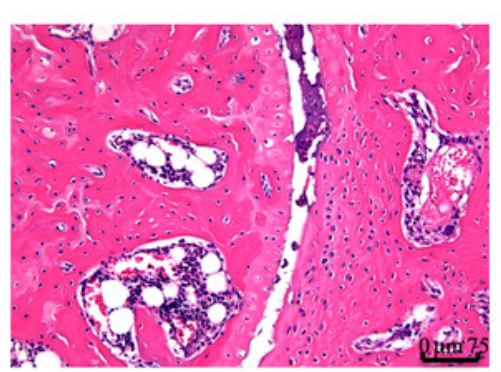

B

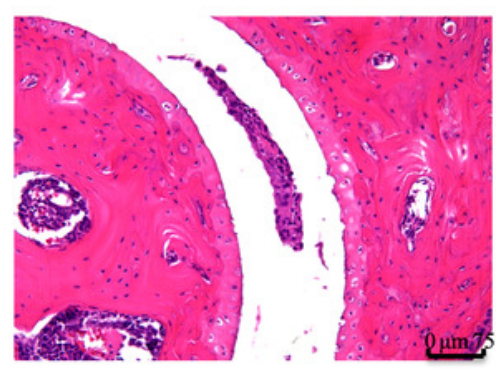

D

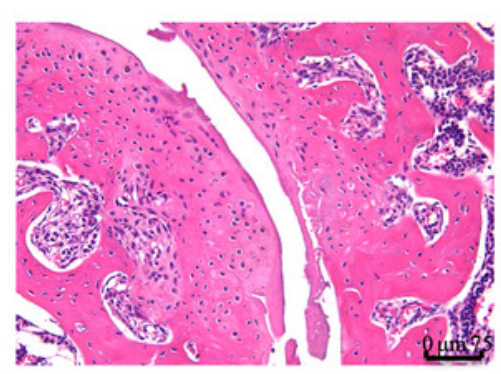

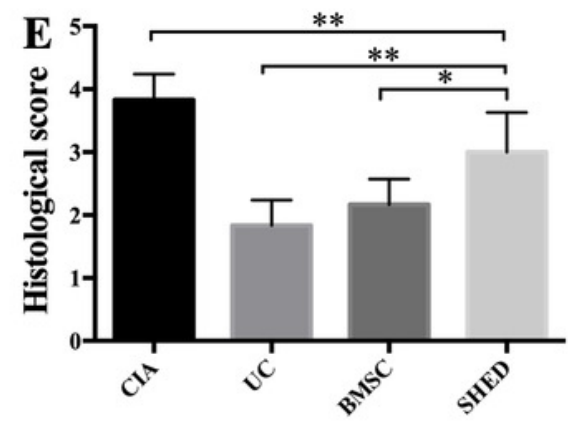




\section{Figure 5}

MSCs reduced inflammatory cytokine expression in serum andhind limb ankle joins.

Level of serum total TNF- $\alpha(A)$ and IL-1 $\beta$ (B) was measured by ELISA assay. MSC treatment reduced expression of TNF- $\alpha$ and IL-1 $\beta$.The expression of TNF- $\alpha$ and IL-1 $\beta$ decreased in UC compared to BMSC and SHED, while the expression was lower in BMSC compared to SHED.Representative images of immunoreactivity for TNF- $\alpha(C, D)$ and IL-I $(E, F)$ in hind limb ankle joins of the mice. The expressions of TNF- $\alpha$ and IL-1 $\beta$ decreased in MSC treatment groups compared with CIA group, and UC group showed lower TNF- $\alpha$ and IL-1 $\beta$ compared to BMSC or SHED group, while the expression was lower in BMSC compared to SHED. The histological score of each group was evaluated and presented in column. Scale bar, $75 \mu \mathrm{m}$. The data indicate the mean \pm SD of six mice per group from three independent experiments. The data were analyzed using one-way ANOVA for comparison among multiple groups and Tukey's multiple comparisons test for comparison between two groups $(* \mathrm{P}<0.05$, $* * \mathrm{P}<$ $0.01, * * * \mathrm{P}<0.001)$. 

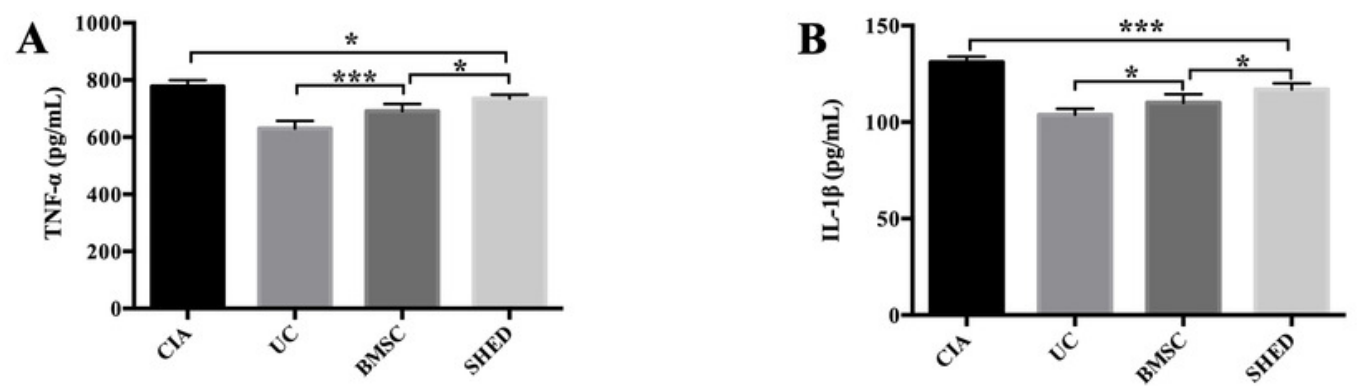

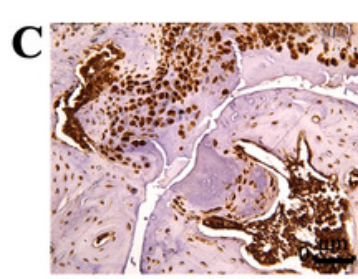

CIA

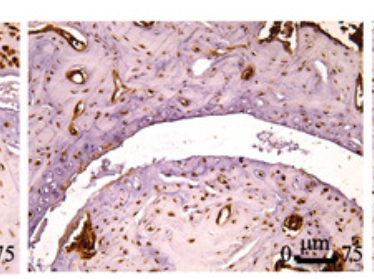

UC

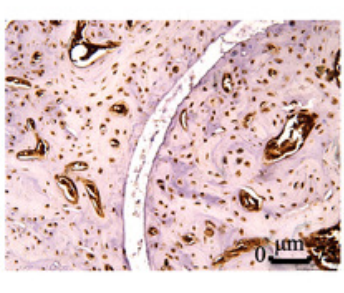

BMSC

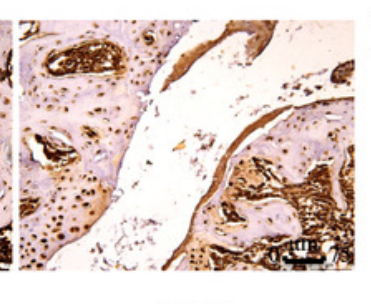

SHED

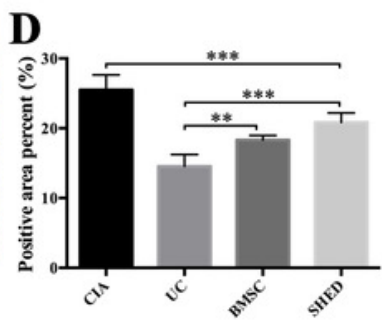

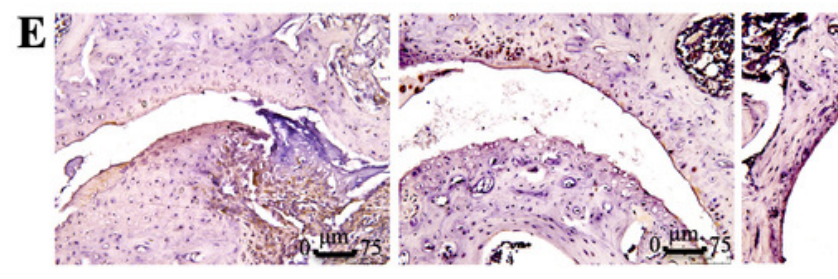

CIA
UC
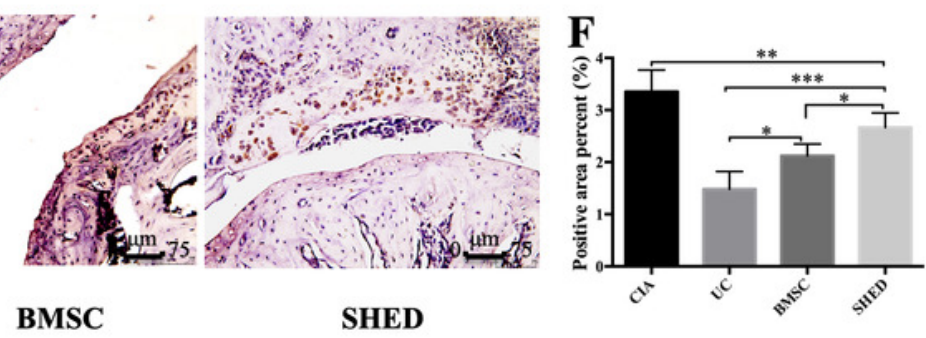